\title{
Study of Sweep and Induced Dihedral Effects in Subsonic Axial Flow Compressor Passages-Part II: Detailed Study of the Effects on Tip Leakage Phenomena
}

\author{
P. V. Ramakrishna and M. Govardhan \\ Thermal Turbomachines Laboratory, Department of Mechanical Engineering, Indian Institute of Technology Madras, \\ Chennai, 600036, India \\ Correspondence should be addressed to M. Govardhan, gova@iitm.ac.in
}

Received 11 September 2009; Revised 3 November 2009; Accepted 21 January 2010

Academic Editor: Chunill Hah

Copyright ( $) 2010$ P. V. Ramakrishna and M. Govardhan. This is an open access article distributed under the Creative Commons Attribution License, which permits unrestricted use, distribution, and reproduction in any medium, provided the original work is properly cited.

\begin{abstract}
This article presents the detailed study of rotor tip leakage related phenomena in a low speed axial compressor rotor passages for three sweep configurations [Unswept (UNS), Tip Chordline Swept (TCS) and Axially Swept (AXS)]. Fifteen domains are numerically studied with 5 sweep configurations $\left(0^{\circ}, 20^{\circ} \mathrm{TCS}, 30^{\circ} \mathrm{TCS}, 20^{\circ} \mathrm{AXS}\right.$, and $\left.30^{\circ} \mathrm{AXS}\right)$ and for 3 tip clearances $(0.0 \%$, $0.7 \%$ and $2.7 \%$ of the blade chord). Results were well validated with experimental data. Observations near the tip reveal that UNS rotor shows high sensitivity than the swept rotors in the blade pressure distribution with change in tip clearance. AXS rotor has high loading capability and less tip clearance effect on blade loading at the near stall mass flow. Downstream shift of the vortex rollup along the chord is observed with increased flow coefficient and increment in the tip gap height. In particular, the effect of flow coefficient is more predominant on this effect. Tip vortex-related flow blockage is less with the swept rotors. Among the rotors, the AXS rotor is found to incur low total pressure losses attributable to tip leakage. Effect of incidence is observed on the flow leakage direction.
\end{abstract}

\section{Introduction}

This paper presents the results of a detailed study conducted to investigate various parameters affecting tip leakage phenomena, including the effect when the rotor is given two varieties of forward sweep. In the first part (Ramakrishna and Govardhan [1]) some fundamental differences a forward sweep brings to the compressor flow pattern (on incidence, deflection, and streamline curvature; and to some extent, effect on the succeeding radial stator) were described. A little on some global performance parameters was also discussed (total pressure rise and stagnation loss coefficient). In this paper, more insight on the internal flow physics, especially tip leakage phenomena with the three sweep configurations (UNS, TCS, and AXS), is presented. Background to this work, computational formulation, validation and so forth, have been presented in Part 1 and so will not be repeated here. The effects of sweep and tip clearance on the overall performance of the stage from the present study are presented in detail in Ramakrishna and Govardhan [2].

Literature available on the effect of tip clearance in swept rotors to assess the same for various sweep configurations is not substantial. Notable works on this subject are of: Gallimore et al. [3], in which they studied both sweep and dihedral effects through low-speed multistage models for a given constant tip gap. The application of forward sweep was disappointing near the casing, where tip clearance loss was increased. On the other hand, positive dihedral reduced tip clearance and hub corner losses. McNulty et al. [4] studied two varieties of tip clearance environments, one with strong tip leakage flows and the other with more moderate levels, for a given sweep configuration. Forward swept blades demonstrated improvements in stall margin, efficiency, and clearance sensitivity when compared to the conventional radial blade. Reduced blade loading near the tip is observed with swept blades which resulted in lower tip clearance flow 
blockage. Both the above mentioned studies were carried out in low-speed compressor environment.

To the best of the authors' knowledge, the present work is unique to the open literature for the reason that this work assesses the combined effects of sweep and tip clearance for various sweep and tip clearance configurations in detail. Conclusions are drawn based on the trends so seen from the different sweep versus tip gap configurations matrix. Though five different sweep configurations were studied (UNS, $20^{\circ} \mathrm{TCS}, 30^{\circ} \mathrm{TCS}, 20^{\circ} \mathrm{AXS}$, and $30^{\circ} \mathrm{AXS}$ ) for the present investigation, only the results with UNS, $20^{\circ} \mathrm{TCS}$, and $20^{\circ}$ AXS rotor configurations are presented for the sake of brevity. The candidate rotors $20^{\circ}$ TCS and $20^{\circ}$ AXS are representatives of their corresponding sweep configurations. Studies have been conducted for three tip clearance configurations: zero clearance, $0.5 \mathrm{~mm}$, and $2.0 \mathrm{~mm}$ tip gaps $(\tau / \mathrm{ch}=0.0 \%, 0.7 \%$ and $2.7 \%)$ and the same are presented in this paper.

\section{Observations}

2.1. Static Pressure Distribution on the Blade. Rotor blade loading is a key feature particularly near the tip, which deserves careful observation and study since (1) local blade loading near the tip TE determines the onset of suction endwall flow separation and stall cell formation [5], while it determines tolerance of the aerofoil to changes in the flow incidences near the tip LE (operating range), (2) local pressure difference between PS and SS near the tip influences the extent of leakage and its velocity (loss) and (3) trajectory of the leakage vortex thus forth driven determines the extent of blockage, which in turn determines the pressure developed in the passage (on pressure rise). Focus is now made on each of these concerns to assess how rotor blade loading near the tip is influenced by sweep and tip gaps.

2.1.1. Static Pressure Distribution around the Blade. Figures 1,2 , and 3 show static pressure distribution on the blade surface for the three rotors at $\phi=0.50$, which is the near stall flow coefficient. In Figures 1(a), 2(a), and 3(a), each graph is plotted for the three rotors for a given clearance. Figures 1(b), 2(b), and 3(b) show the same graphs, plotted for each rotor for different clearances. Evaluating these graphs sideby-side gives better understanding. These graphs are plotted at spanwise locations $\left(S_{1}\right) 1.5 \mathrm{~mm},\left(S_{2}\right) 10 \mathrm{~mm}$, and $\left(S_{3}\right)$ $20 \mathrm{~mm}$ from the rotor tip for various clearances. Note that $S_{2}$ corresponds to the location which is at a distance 20 times the physical tip gap height from the rotor tip with reference to the clearance $\tau=0.5 \mathrm{~mm}$ and 10 times with reference to $\tau=2 \mathrm{~mm}$. Similarly $S_{3}$ refers to the location at 40 times and 20 times the respective physical tip gap heights. Since these locations are at different distances from the outer casing wall for the different tip gap heights, effect of endwall and blade surface boundary layer is embedded in these results. However, as the primary intention is to observe the flow pattern for different cases, this boundary layer influence is not separately assessed.
At Location $S_{1}$ ( $1.5 \mathrm{~mm}$ from the Tip). At all clearances, high suction peak is found for the UNS rotor. Flattening of static pressure distribution near the pressure surface LE can be observed with increasing clearance due to the influence of "vena-contracta," described through Figure 4, which shows the tip surface static pressure distribution contours at the two nonzero clearances. From about $15 \%-30 \%$ of the chord, zones of low pressure at the pressure surface edge of the tip are observed. This low pressure is due to flow taking high curvature while entering the tip gap. When the clearance is increased, differences in the pressure surface static pressure distributions of all the rotors are minimizing while suction peak moves towards downstream of the chord. Steadiness of blade loading with increasing clearance for different rotors can be evaluated from Figure 1(b). Static pressure distribution of AXS rotor is more stable than the other rotors when clearance is increased. UNS rotor shows very high sensitivity to the tip clearance flows for two reasons that at this mass flow, this rotor was already found to be stalled; and the severe separation has occurred near the suction surface blade tip, where the pressure distribution is plotted.

At Location $S_{2}$ (10 mm from the Tip). "Flattening" effect near the pressure surface LE is not observed with $\tau / \mathrm{ch}$ $=0.7 \%$, while a little shift is observed with $\tau / \mathrm{ch}=2.7 \%$ configurations. This means that on pressure surface, the effect of vena contracta on blade static pressure distribution is slightly felt even till a radial extent 10 times the clearance gap height, while it is totally disappeared at 20 tip gap heights. At $\tau / \mathrm{ch}=2.7 \%$, all the three rotors had overlapping pressure distributions. The deep negative pressures on suction surface (due to tip vortex), though reduced, yet are observed for both the clearances. This implies that the effect of leakage vortex is still felt even at a radial extent 20 times the gap height on the suction surface pressure distribution. As a general observation, increased clearance makes suction surface pressure distribution more negative. This is gradually felt by the UNS rotors and very less felt by the AXS rotor. TCS rotor showed suddenly raised negative pressures on the suction side when the clearance is increased to maximum. For the initial increment of the tip gap $(\tau / \mathrm{ch}=0.0 \%$ to $\tau / \mathrm{ch}=0.7 \%)$, pressure distribution is not much altered. This is observed at all the three radial locations $\left(S_{1}, S_{2}\right.$, and $\left.S_{3}\right)$. This hints that with TCS rotors, strength of the leakage vortex does not follow the same function of tip gap height as it does with other sweep configurations.

At Location $S_{3}$ (20 $\mathrm{mm}$ from the Tip). The effect of clearance flow on the pressure distribution is almost stalled at this location. Through the circumferential and radial extents of leakage vortex blockage, Suder and Celestina [6] observed that influence of the tip leakage flow is seen over radial extent 20 times the physical clearance height in transonic compressor rotors. This is in agreement with the current results pertaining to subsonic compressor environment as well. 

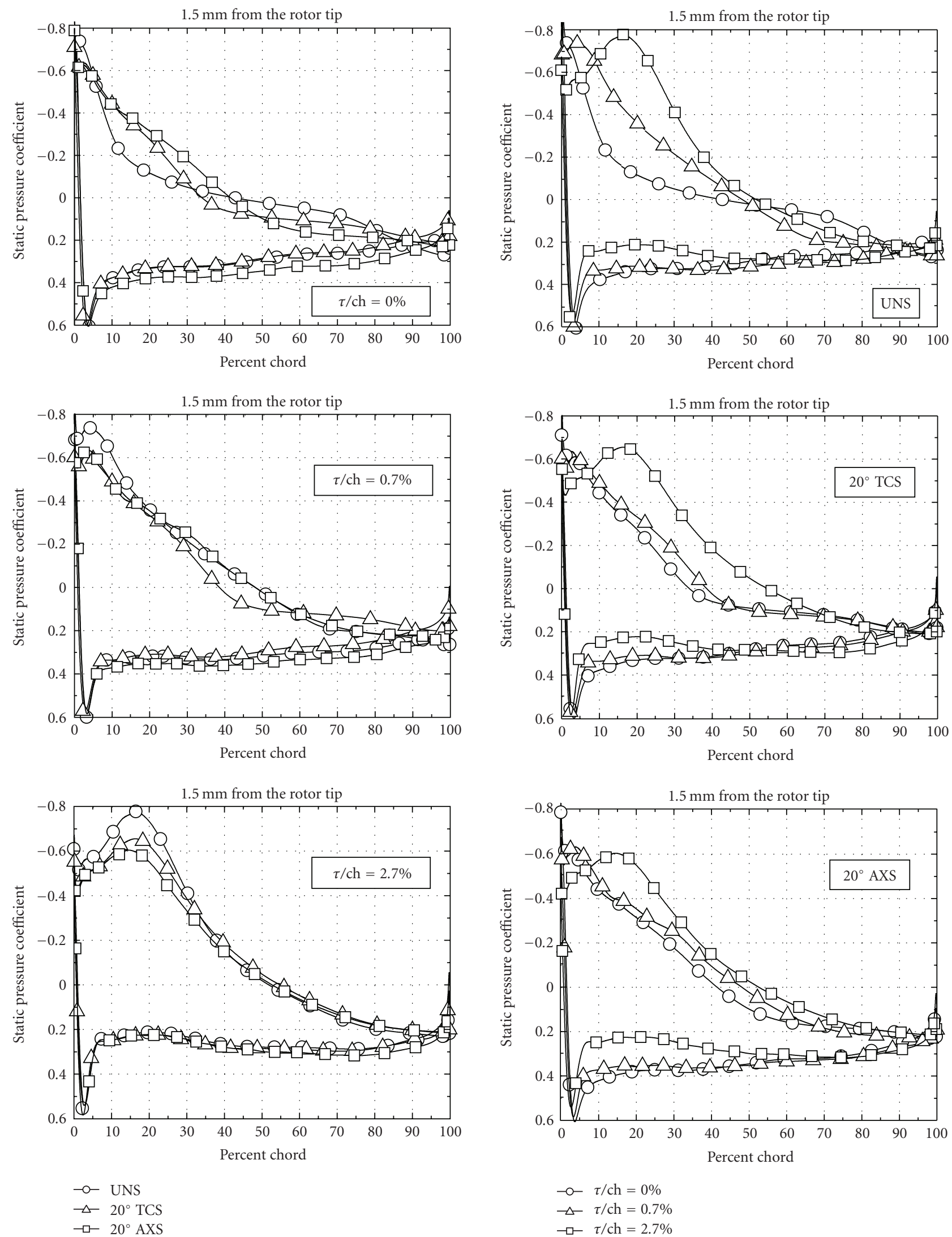

(a)

(b)

FIgURE 1: Static pressure distribution on the blade at $S_{1}, \phi=0.50$. 

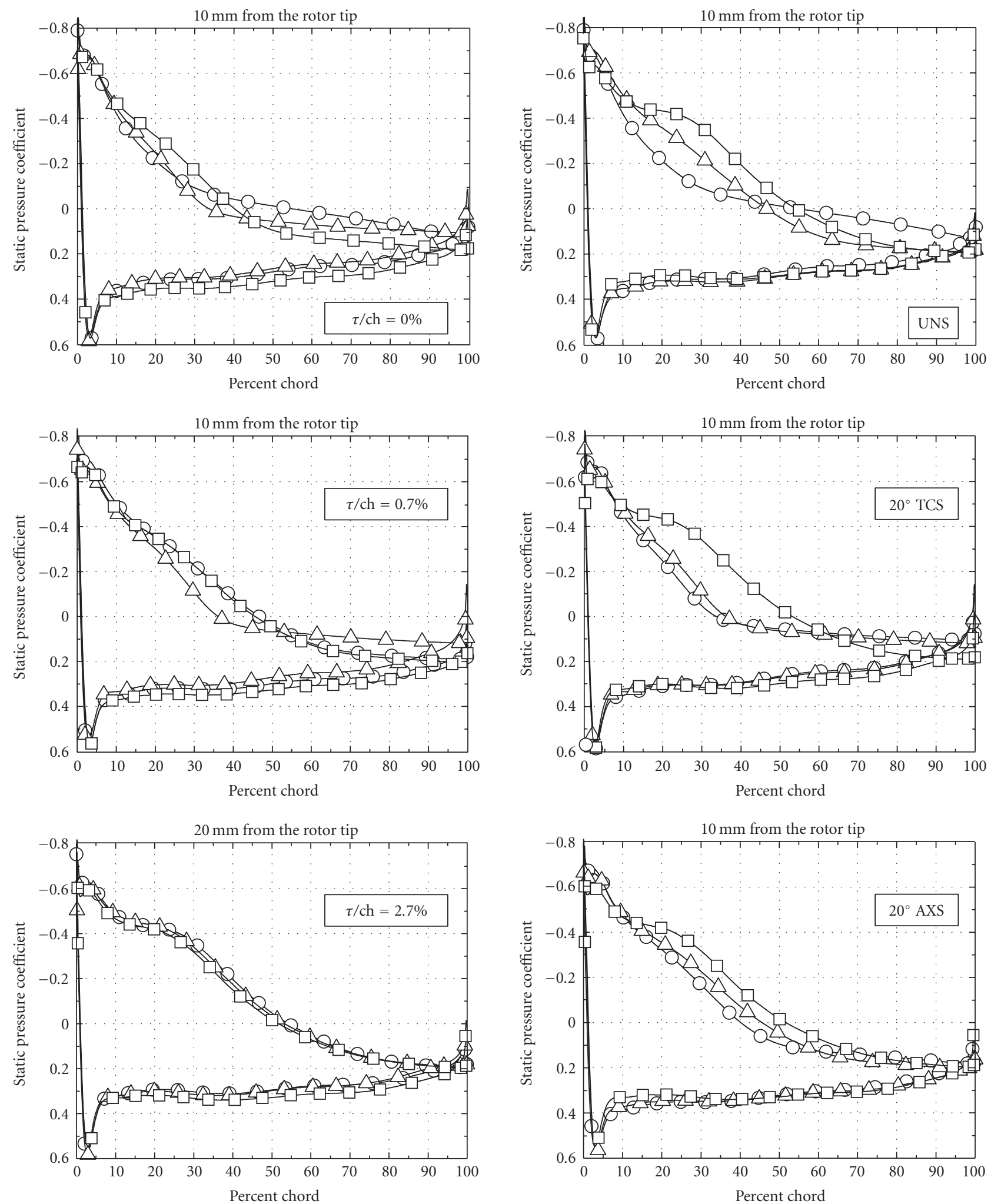

$$
\begin{aligned}
& \multimap-\text { UNS } \\
& \triangle \quad 20^{\circ} \text { TCS } \\
& \square-20^{\circ} \text { AXS }
\end{aligned}
$$$$
\begin{aligned}
& -\_\tau / \mathrm{ch}=0 \% \\
& -\tau / \mathrm{ch}=0.7 \% \\
& \square-\tau / \mathrm{ch}=2.7 \%
\end{aligned}
$$

(a)

(b)

Figure 2: Static pressure distribution on the blade at $S_{2}, \phi=0.50$. 

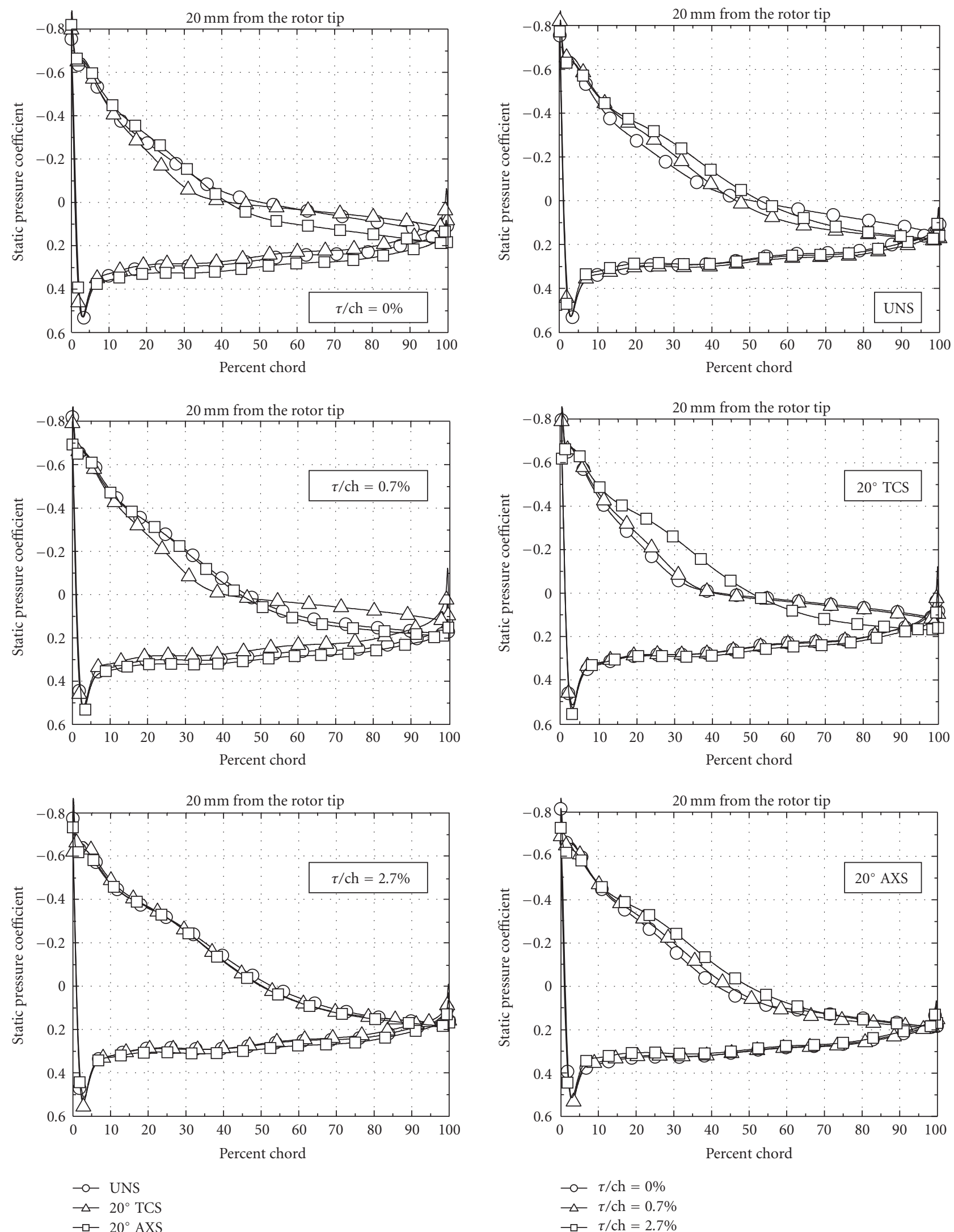

$$
\begin{array}{ll}
\multimap & \tau / \mathrm{ch}=0 \% \\
\triangle & \tau / \mathrm{ch}=0.7 \% \\
\rightarrow-\tau / \mathrm{ch}=2.7 \%
\end{array}
$$

(a)

(b)

FIgURE 3: Static pressure distribution on the blade at $S_{3}, \phi=0.50$. 


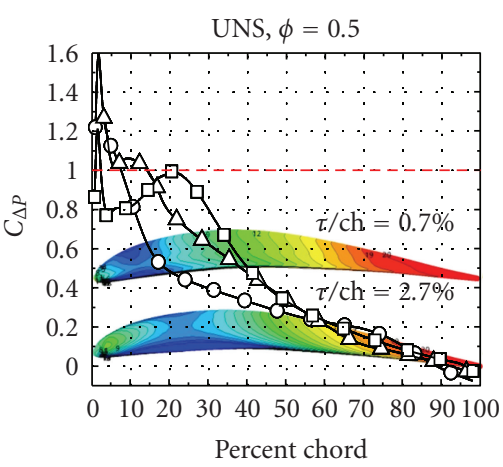

(a)

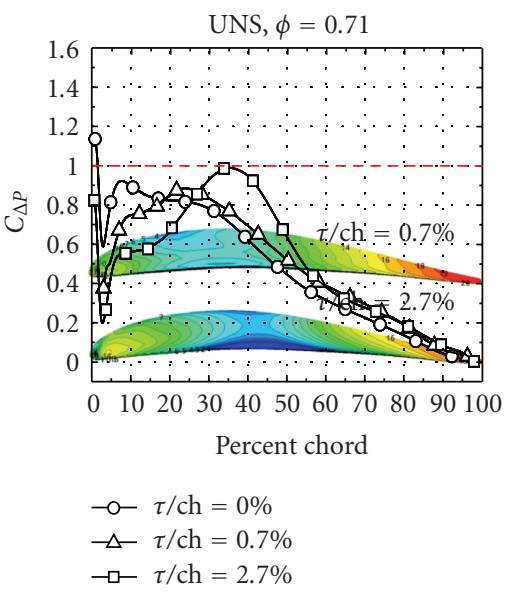

(d)

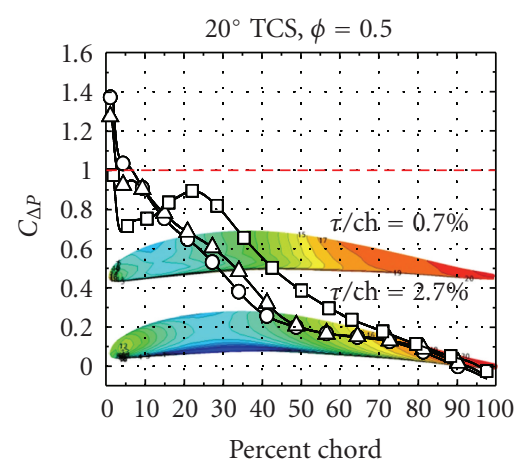

(b)

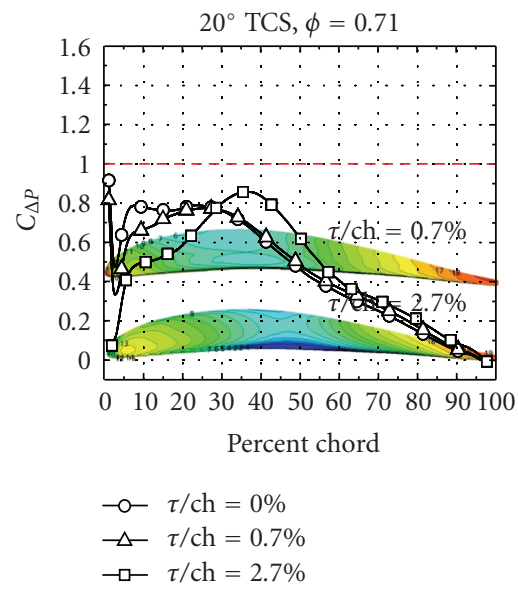

(e)

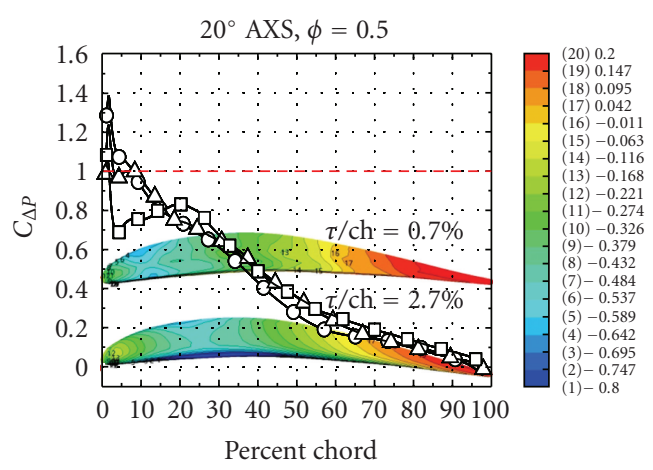

(c)

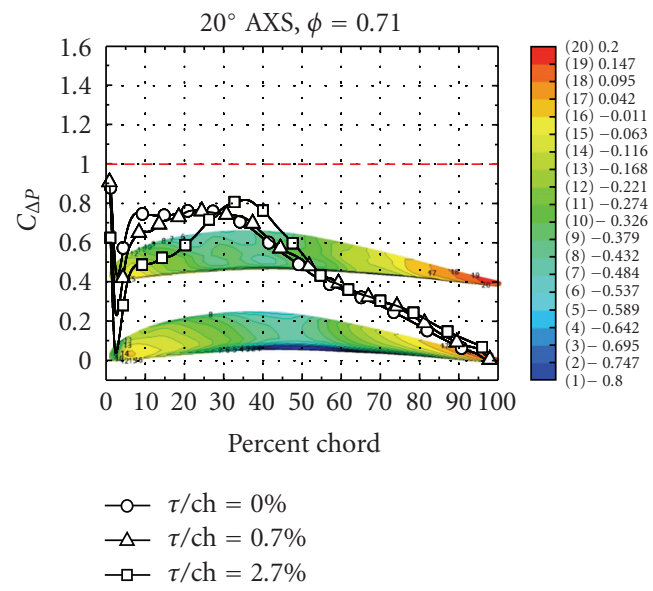

(f)

Figure 4: Static pressure differential, $C_{\Delta P}$.

2.1.2. Local Pressure Difference between PS and SS near the Tip. Govardhan et al. [7] observed the blade loading to decrease substantially just under the blade tip surface. The effect was found to be pronounced when the tip clearance is increased. Static pressure differential, $C_{\Delta P}$, is defined as the difference of static pressure coefficient on the pressure and suction surfaces of the blade, in such a way that differencing is done approximately perpendicular to the camber line. This is a direct indication of the degree of blade loading along the chord. Tip surface static pressure distribution contours are plotted behind the $C_{\Delta P}$ variation graphs for clarity:

$$
C_{\Delta P}=\left[\psi_{s}\right]_{\mathrm{PS}}-\left[\psi_{s}\right]_{\mathrm{SS}} .
$$

Variation of $C_{\Delta P}$ is measured at a radial position $1.5 \mathrm{~mm}$ beneath the rotor tip (Figure 4). At low flow coefficient, compared to the other rotors, higher loading is observed for UNS rotor up to $20 \%-30 \%$ of chord from the LE (till the negative suction peak zone), depending upon the clearance. Reduced blade loading of UNS rotor at $\tau / \mathrm{ch}=0.0 \%$ is because of the flow blockage due to violent flow separation on the suction surface from much of the blade region near the tip (more evident from Figure 5). Otherwise, as a general observation, high loading is seen for the rotors in the order UNS, $20^{\circ} \mathrm{AXS}$, and $20^{\circ} \mathrm{TCS}$ near tip-leading edge portions. $20^{\circ}$ AXS rotor is found to have high blade loading on the rest of the blade. Sections 2.1.1 and 2.1.2 infer that AXS rotor has high loading capability and less tip clearance effect on blade loading at the near stall mass flow. It is clear that high variations in loading distribution with change in tip gap are seen for UNS rotor. TCS rotor showed sudden shift in the curve when the clearance is increased from $\tau / \mathrm{ch}=0.7 \%$ to the maximum clearance. AXS rotor distribution is least altered with the clearance changes.

These observations are similar at high flow coefficients as well. UNS rotor exhibited higher loading along the chord till the negative suction peak zone. It is clear that this negative suction peak is shifted to downstream when the mass flow rate is increased. This peak corresponds to the lowest static pressure trough seen on the rotor tip pressure distribution contours, which is caused by the leakage flow taking high curvature while entering the tip gap and forcibly spilling out to the other side through the gap. This is a "thoroughfare zone" for the tip leakage flow, at the end of which originates the leakage vortex.

2.1.3. Flow Blockage. In order to comment on flow blockage caused by the tip leakage flows, leakage vortices and the flow separations existing around the blade, blade-to-blade contours of axial velocity coefficient $\left(C_{m} / U_{t}\right)$, are plotted for $\phi=$ 0.50 in Figure 5 on planes $1.5 \mathrm{~mm}$ underneath the rotor tip. 


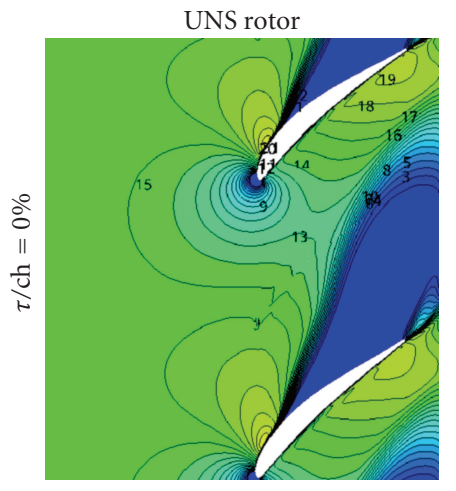

(a)

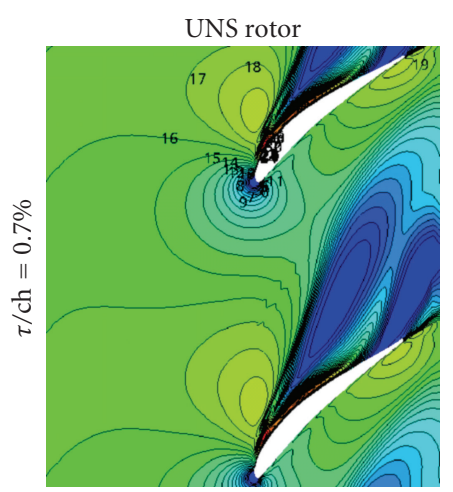

(d)

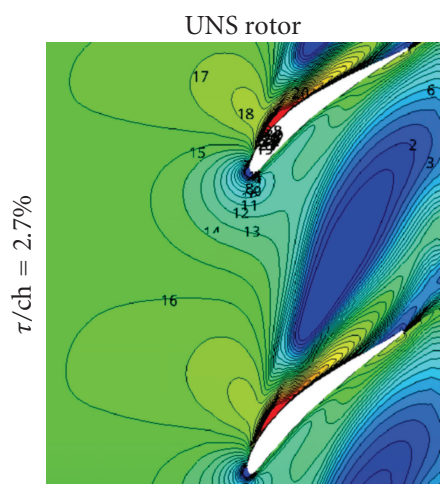

(g)

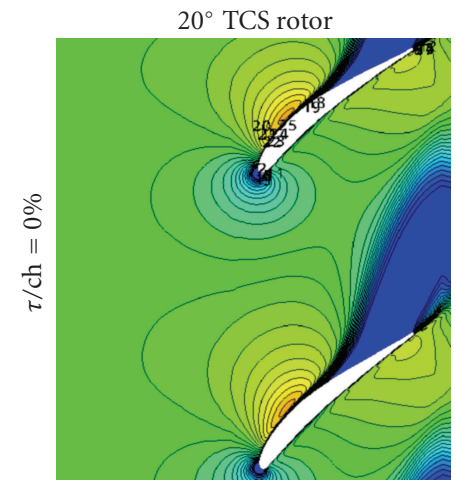

(b)

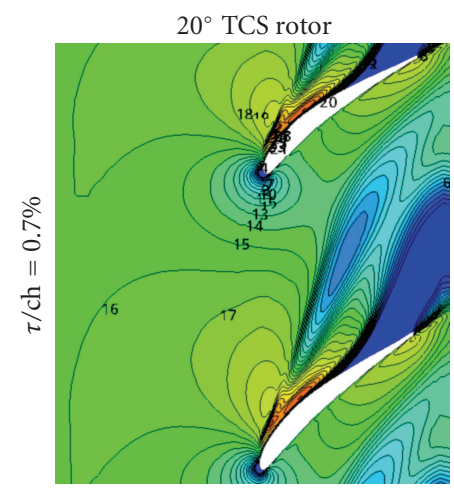

(e)

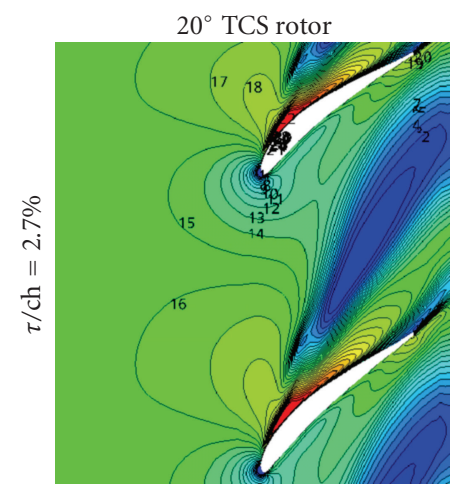

(h)

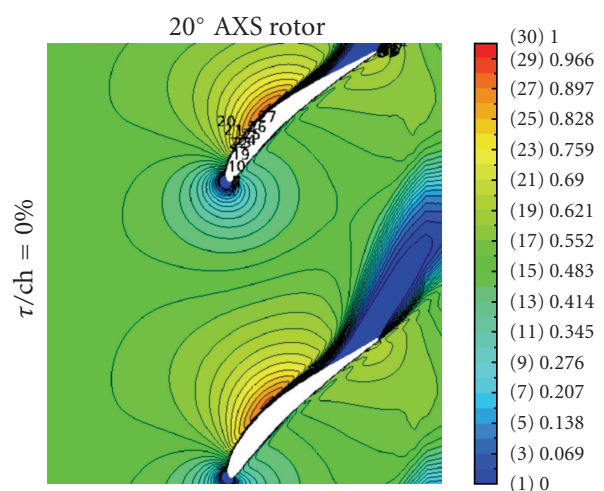

(c)

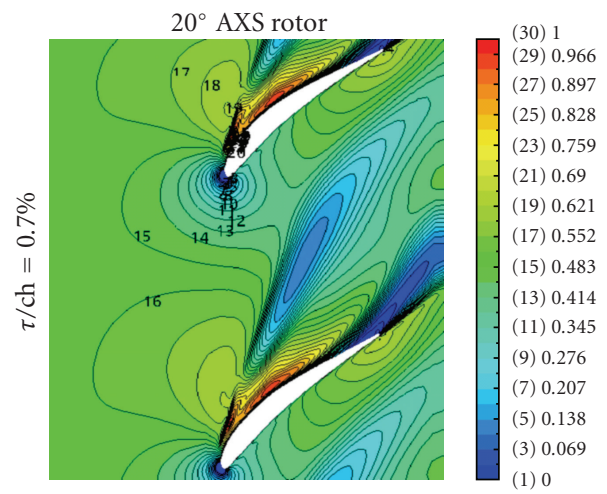

(f)

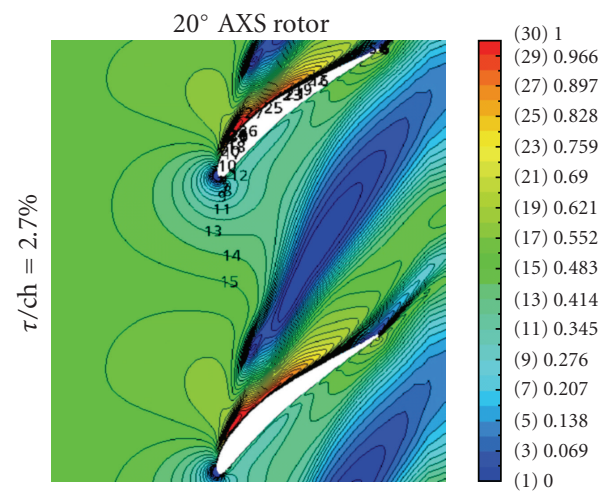

(i)

Figure 5: Axial velocity coefficient contours on blade-to-blade planes $1.5 \mathrm{~mm}$ from the rotor tip at $\phi=0.50$.

In the contours, flow blockage is attributed to the regions with zero or negative axial velocity coefficient. This implies that blockage here means the mechanism which essentially deprives a fraction of passage area being useful for the flow progression, or a flow reversal. It is evident that at $\tau / \mathrm{ch}$ $=0.0 \%$, there is huge flow blockage area spreading over from the LE of suction tip seen for UNS rotor. The severe separation of flow as a result of tip corner wall stall is seen by the thick dark regions, spread over $75 \%$ of the passage area causing enormous blockage. As there is no tip gap, leakage flow contribution to this flow reversal in this case is nil. It is the separation of the suction surface-stagnated fluid, all migrated and accumulated at the tip corner, that is the main cause for this blockage. It can be seen that wake-related low axial velocity region near the TE is merged with this in the contour. When the tip clearance is increased to the higher levels, separate blockage regions are observed for leakage vortex and wake-related separation.

At nonzero clearances, much of the leakage flow exits the clearance gap with a negative axial velocity component, creating vortex-related flow blockage. At both nonzero clearance levels, this blockage is the highest for the UNS rotor and the lowest for the AXS rotor. UNS rotor leakage vortices penetrated more into the passage before the axial velocity 


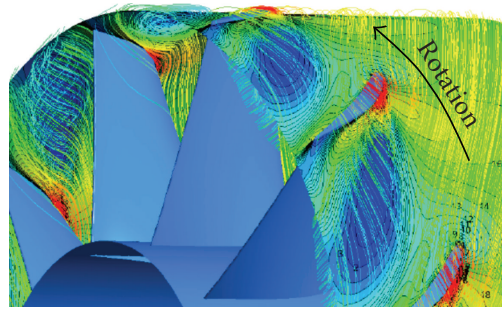

FIGURE 6: Flow scenario with leakage flow, UNS rotor $\tau / \mathrm{ch}=2.7 \%$.

deficit get sufficiently dissolved. This problem is found to be less for the swept rotors. In the case of TCS rotor, at zero clearance, the separation is less severe when compared with the UNS rotor. When the clearance is increased to $\tau / \mathrm{ch}=0.7 \%$, the separation phenomena near the TE is less influenced by the tip leakage and hence equally severe. In addition to this, as the leakage flow has its own detrimental effects on flow, $20^{\circ}$ TCS rotor is stalled (observed on the pressure characteristic) at this flow coefficient with $\tau / \mathrm{ch}=$ $0.7 \%$, with regard to these two reasons. When the tip gap is increased to $\tau / \mathrm{ch}=2.7 \%$, reduced wake-related separation can be seen from the contour, damped by the tip leakage flow. AXS rotor has less tip corner separation at zero clearance and the twofold flow blockages are also less severe with higher tip clearances. A typical "flow blockage" due to tip leakage vortex is shown in Figure 6 which corresponds to the case of UNS rotor with $2.7 \%$ clearance (Figure $5(\mathrm{~g})$ ) at $\phi=0.50$. Flow leaking from the tip gap demonstrates the relative velocity variations. Maximum velocity can be seen for those streamlines rolling through the lower static pressure "thoroughfare zone" seen in Figure 4.

2.2. Tip Leakage Phenomena. The same flow scenario is shown in Figure 7 with relative velocity contour plotted on blade-to-blade plane, 3D velocity streamlines leaking through the tip gap and static pressure distribution contour on the rotor tip. Contour limits and intervals for all the relative velocity contours shown henceforth are the same as those presented in Figure 7. Streamlines crossing the tip gap through the low static pressure zone inside the tip have highest leakage velocity. This figure admits that tip leakage flow is fully $3 \mathrm{D}$ in nature, which is neither normal to the camber line [8] nor parallel to the rotation (tangential). They flow approximately tangentially near the blade TE, where the pressure differentials between PS and SS are the lowest on the chord.

A downstream shift of the vortex rollup along the chord is observed with increased flow coefficient and increase in the tip gap height. In particular, the effect of flow coefficient is more predominant on the location of the pressure trough. This shift has been reported in the literature, but the reasons were not well explained. Possible factors affecting this shift include local flow incidence and tip aerofoil loading. It has been reported in the first part of this paper [1] that AXS rotor received the flow at lower incidence near the tip sections. Local incidence is the highest for the UNS rotor and moderate for TCS rotor. But the extent of incidence effect in shifting this vortex rollup to downstream chordwise portions is not clear from the graphs (Figure 4). All the three sweep configurations showed similar chordwise location for the $C_{\triangle P}$ peak. On the other hand, at lower flow coefficients, due to higher blade loading conditions, the leakage flow would quickly tend to roll up into vortex resulting in early portion of the tip chord subjected to lower pressure troughs where a strong vortex is originated. The same would shift to downstream chordwise locations with increase in flow coefficient where blade loading conditions are relatively weaker. Nevertheless, if the magnitude of $C_{\Delta P}$ is observed, at the higher clearance $\tau / \mathrm{ch}=2.7 \%$, the peak value is found to be the same at both minimum and maximum flow coefficients. That is, though downstream shift of this low trough zone is observed with increased mass flow rate, value of peak $C_{\Delta P}(\approx 1$ for UNS rotor $)$ remained the same. This is, as a general observation, found with all the three sweep configurations; however, it is not observed for the $\tau / \mathrm{ch}=$ $0.7 \%$ clearance case.

When a small clearance is introduced to a zero-tip clearance rotor by suddenly cutting some portion of the blade, flow would swiftly leak owing to the local blade loading conditions. This causes pressures on the suction edge to drop further, leading to much higher pressure differentials having potential to draw some more leakage flow. This process eventually stabilizes at certain leakage conditions, which are case specific. The rolling vortex causes flow blockage in the upstream portions of the passage which results in slightly increased incidence. Now, if the blade element is again cut at the tip to further increase the clearance, it would lead the leakage flow pattern to shift to another set of definite conditions. If the latter results in shifting the pressure trough to downstream chord portions, it must be the increased pressure differential (further) across the tip gap and downstream shifted $C_{\triangle P}$ peak which would lead the leakage conditions to shift to a pattern where the leakage vortex rollup is moved to the downstream chord. At a small tip clearance $(\tau / \mathrm{ch}=0.7 \%)$, rotors did not show any constancy in the peak $C_{\Delta P}$ value with increasing flow coefficient, which indicates that the leakage did not "stabilize" for the local aerofoil loading, which is the case when the clearance is further increased to $\tau / \mathrm{ch}=2.7 \%$.

Storer and Cumpsty [9] derived expressions for component of leakage velocity in the streamwise direction $\left(C_{S}\right)$ and absolute leakage velocity $(C)$ based on appropriate assumptions. Then the component of leakage velocity normal to the camber line $\left(C_{L}\right)$ is derived as $C$ is resolved into mutually perpendicular components $C_{S}$ and $C_{L}$. This philosophy indicates that the least is the streamwise pressure gradient (on the pressure side) at the entry to the tip gap, the more normal is the leakage flow to the camber line. When the mass flow rate is reduced, streamwise pressure gradients increase faster than the blade loading. It follows that at a low flow coefficient, the tip flow leaks more tangentially than at higher flow coefficients, but the opposite is observed in the present study. Hence it is expected that the higher streamwise pressure gradient which is adverse in nature would contribute, to some extent, in picking the chordwise location for the pressure trough, but its influence 


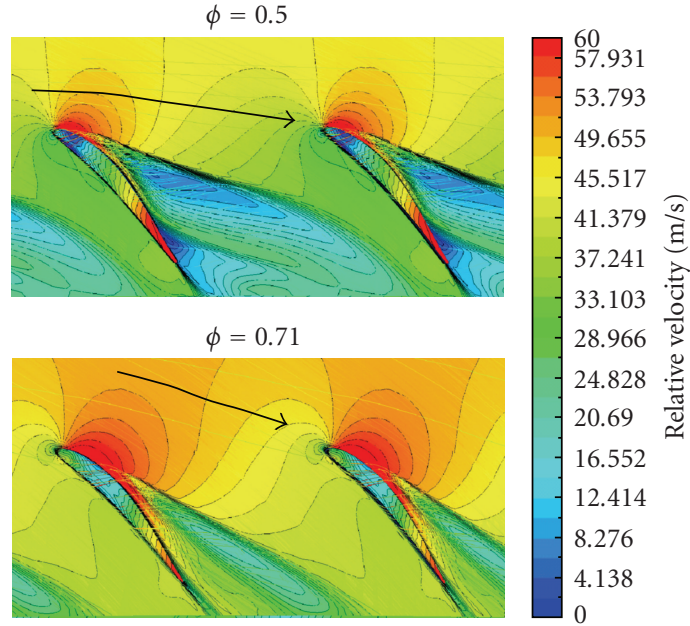

(a) $\tau / \mathrm{ch}=0.7 \%$

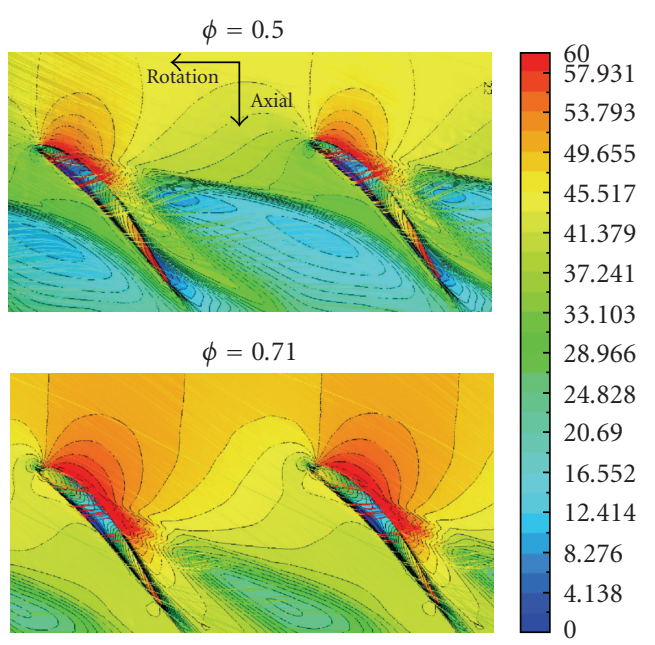

(b) $\tau / \mathrm{ch}=2.7 \%$

FIGURE 7: Flow scenario for UNS rotor near the tip showing leakage flow, blockage with leakage vortex, and wake effect.

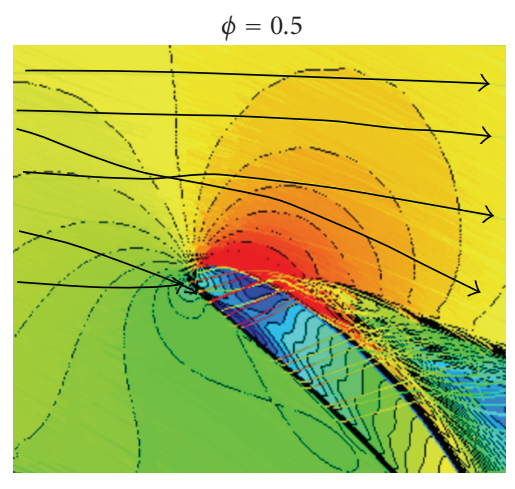

(a)

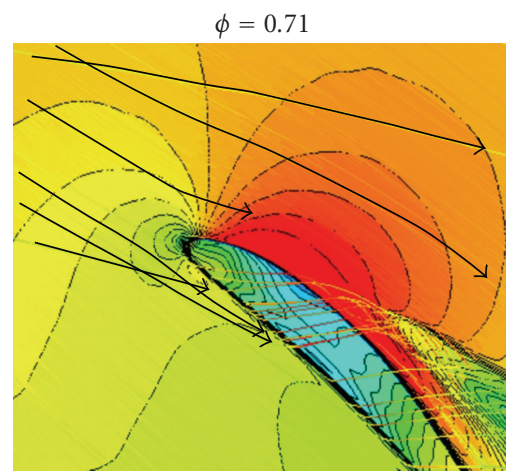

(b)

FIgURE 8: Flow scenario near front portions of the chord, UNS, $\tau / \mathrm{ch}=0.7 \%$.

on the leakage flow direction is less obvious. Increased mass flow (high inlet velocity) brings higher streamwise flow velocity at the tip gap entrance. This, accompanied by the low adverse pressure gradients, would invariably delay the vortex rollup at higher flow coefficients. Hence, high incidence and peak $C_{\Delta P}$ close to the LE portion of the chord (at zero clearance) accompanied by more streamwise adverse pressure gradients are observed to be the primary reasons for early leakage at low flow coefficients. It is to be noted that though some comments are made on the individual effect of these parameters on the leakage pattern, it is not legitimate to look at a combined effect and derive conclusions on the individual causes. For example, at high flow coefficient, there is a region of chord (about 30\% front chord) over which the pressure differential $C_{\Delta P}$ is observed to be continuously high (Figure 4 ). Together with the lower flow incidence at this flow coefficient, the tip flow became less normal to the camber line (more tangential) and the pressure trough is shifted to the downstream chord locations. The flow scenario near front portions of the chord, along with the pressure trough and the relative streamline trend (showing incidences), is shown in Figure 8 for better illustration. Figure 8 is the magnified version of Figure 7. This confirms the definite influence of the incidence angle on the leakage pattern when it is accompanied by the other factors.

Relative velocity contour describes the leakage vortex penetration. It is discussed in the previous sections that among the rotors, the tip leakage vortex in the UNS rotor penetrated more into the passage, almost till the pressure surface of the adjacent blade with the highest axial velocity deficits. In Figure 7, clear cut lines of the relative velocity contour demarcating the higher flow reversal regions from the main flow can be observed. It is seen that for this UNS rotor case at $\phi=0.50$ and $\tau / \mathrm{ch}=2.7 \%$, where the leakage vortex penetration and blockage are the highest among the various clearance and rotor configurations, the relative velocity deficit region is observed to penetrate close to the TE of the adjacent blade, without crossing the tip again to cause what is referred to as "double leakage" [10]. Double leakage refers to tip clearance flow leaking across the adjacent blade tip again but with a lower streamwise velocity component. This has not occurred for any case including this most potential case (UNS with $\tau / \mathrm{ch}=2.7 \%$ ). 
2.3. Flow Mixing within the Tip Gap. Flow physics within the tip gap typically depends on local blade thickness to the tip gap height $(t / \tau)$. For instance, if the tip gap is extremely small, tip clearance lies within the boundary layer and the effect caused by the leakage flow is felt through a modification of boundary layer thickness. On the other hand, if the gap is large, it allows higher mass flow to pass through it. For low $t / \tau$ configurations (typically seen in compressors) because of the typical low ratio of blade thickness to the gap height, the leakage jet typically emerges from the gap with a core of inviscid flow, due to incomplete mixing inside the gap. The longer is the blade thickness, the greater is the mixing expected. Heyes and Hodson [11] suggested that mixing inside the tip gap would be complete only if the gap length exceeds six gap heights. Thus, for the short tip gaps, Heyes and Hodson expected a core of loss-free fluid which they refer to as an isentropic jet. Storer and Cumpsty [9] assumed no mixing inside the tip gap. Consequently, as long as the clearance flow spills out without reattaching/mixing inside the tip gap, it would experience no loss in stagnation pressure in passing through the tip clearance (isentropic jet). It follows from Bernoulli's equation that leakage flow and the throughflow would have the same speed, but different directions. On the other hand, Kang and Hirsch [12] in a contemporary study predicted $35 \%-80 \%$ of the total exit loss from the tip gap mainly attributable to mixing. While all these studies pertain to compressors, Moore and Tilton [13] in moderately loaded turbine cascades observed complete mixing inside the tip gap with a thickness-to-tip gap ratio $(t / \tau) \approx 7.3$. They interpreted the flow downstream of venacontracta of the separation bubble as a sudden expansion in flow area, with a consequent loss in total pressure being experienced by the fluid in passing through the tip clearance. However, Govardhan et al. [8] observed incomplete mixing inside the tip gap $(t / \tau=10)$ in a high loaded turbine cascade with a significant loss occurring within the tip gap. Mixing within the tiny tip gap is conclusively a source of loss due to entropy generation and loss in fraction of total pressure, which is typically observed to occur with a $t / \tau$ ratio exceeding 6 . For the present rotor configurations, maximum $t / \tau$ is about 21.5 with $\tau / \mathrm{ch}=0.7 \%$ and about 5.3 with $\tau / \mathrm{ch}=2.7 \%$. Flow mixing is observed even in the latter configuration, all along the chord including the highest $t / \tau(\approx 5.3)$ chordwise location as well as the lowest $t / \tau(\approx 1.41)$ chordwise sections. To look into the flow mixing, endwall and blade tip surface static pressure distributions at various chordwise locations are plotted in Figure 9. The local pitchwise distance is nondimensionalised with the mean chord length. The pressure and suction surfaces are marked in Figure 9.

From the tip gap entrance, the flow experiences contraction in the flow cross-sectional area, hence the static pressure falls rapidly. This is related to vena-contracta. After this sudden contraction, the wall static pressure, going inline with the tip surface static pressure distribution, either remained constant or started recovering from its minimum, depending on the chordwise location and the flow coefficient. Prior to the formation of leakage vortex (at the front portions of the chord), the pressures in either of the walls are deviating towards the tip gap exit. Though sufficiently overlapped pressure distributions are observed within the tip gap, towards the gap exit, different pressures on either of the endwalls are indicative of incomplete mixing, where each pressure distribution when looked separately exhibited its own trend. In the downstream chord portions, after the deep low pressure trough, the pressure distributions on either walls showed a mutual trend (complete mixing). Flow accelerations are very less in these aft portions of the chord, downstream to the tip vortex location $(X / e>20 \%$ at $\phi=0.50$ and shifted to downstream chord at higher flow coefficients: $X / e>32 \%$ at $\phi=0.71)$.

As all these phenomena are found to be qualitatively very much similar with swept rotors as well, plots pertaining to these rotors are not shown here. A quantitative study of flow mixing inside the tip gap is not important to the present perspective study because total pressure loss incurring inside the gap (flow contradicting with isentropic jet behavior) can be better portrayed through total pressure rise contours plotted inside the tip gap.

\subsection{Total Pressure Loss within the Tip Gap. Contours of total} pressure coefficient are plotted in Figure 10. Leakage flow streamlines are also shown colored with relative velocity in these figures for better clarity. These plots are made on pitchwise planes at radial locations passing through the middle of the tip gap. It was already pointed out that there were different estimates on the fractions of loss taking place within the tip gap and after exiting the tip gap. Loss occurring from intense shearing caused by mixing of leakage flow with the main flow which encounter each other at widely different angles is inevitable, whether the flow emerged as isentropic jet or already contributed to some fraction of loss while spilling out through the tip gap. Mixing loss strongly depends on the angle between the mixing jets as well (Storer and Cumpsty [9]).

The high loss core regions are located at the suction edge of the tip, where intense mixing of tip flow with the main flow takes place. The fraction of loss occurring inside the tip gap is comparatively less than that due to this mixing. From the contours, total pressure loss is observed to be the highest for the UNS rotor in all the cases.

2.4.1. At $\tau / c h=0.7 \%$. At low flow coefficient, a fraction of loss has already occurred in passing through the tip gap which is evident from the pressure trough seen on the tip surface. While this zone grew in size at higher mass flow rates, loss due to mixing with the main flow after emerging out shared the higher fraction of loss. The deep low total pressure regions near the tip are observed to increase with flow coefficient. Mixing with the main flow also caused high level of pressure losses because at higher flow coefficients, acceleration on the suction surface-leading edge is also high causing high pressure losses when the same is intensely mixed with the leakage flow. This is observed in the two swept rotors as well. Among the rotors, AXS rotor is found to incur low total pressure losses attributable to tip leakage. 

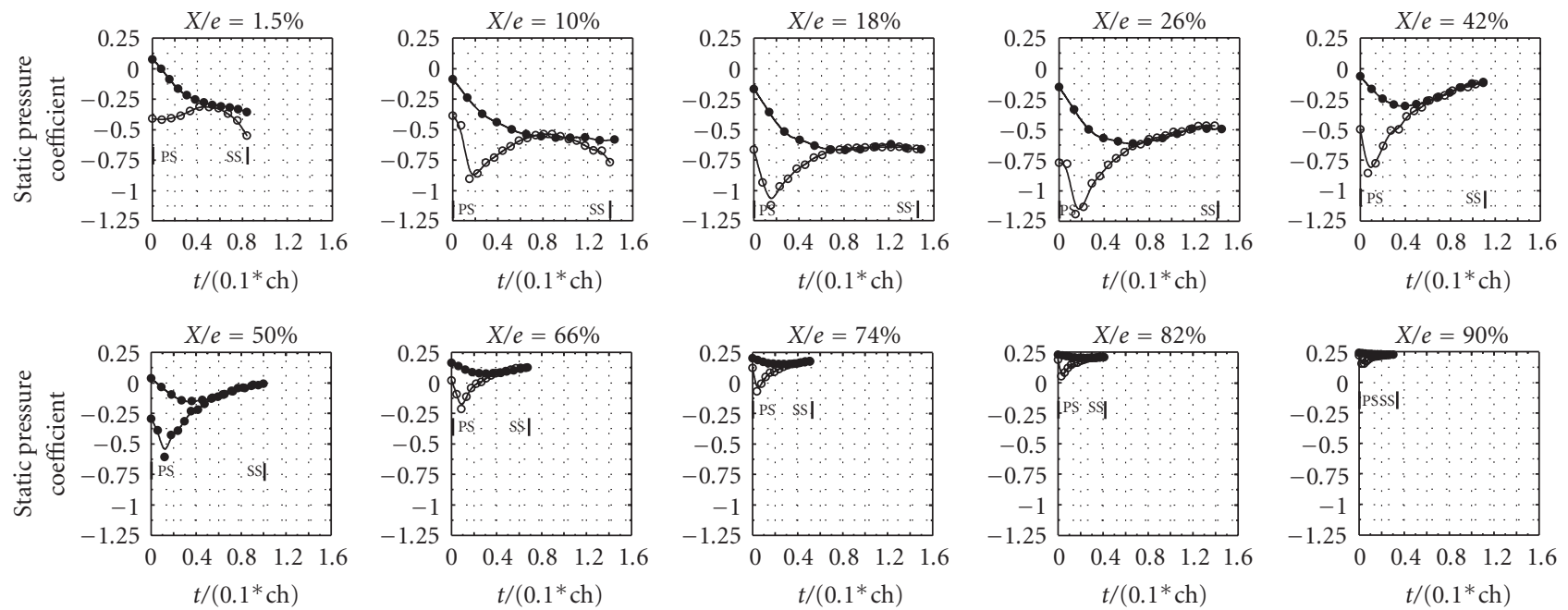

UNS rotor, $\tau / \mathrm{ch}=2.7 \%, \phi=0.5$

$\rightarrow$ Along endwall $\rightarrow$ Along blade tip

UNS rotor, $\tau / \mathrm{ch}=2.7 \%, \phi=0.5$

$\rightarrow$ Along endwall $\rightarrow$ Along blade tip
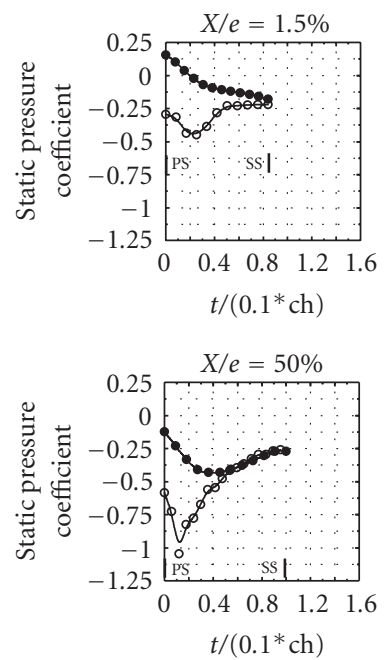

UNS rotor, $\tau / \mathrm{ch}=2.7 \%, \phi=0.71$ $\rightarrow$ Along endwall $\rightarrow$ Along blade tip
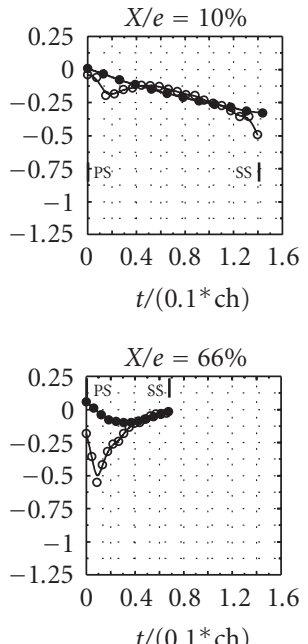

UNS rotor, $\tau / \mathrm{ch}=2.7 \%, \phi=0.71$

$\multimap$ Along endwall $\rightarrow$ Along blade tip
UNS rotor,

$\tau / \mathrm{ch}=2.7 \%, \phi=0.5$

$\rightarrow$ Along endwall

$\rightarrow$ Along blade tip

(a)
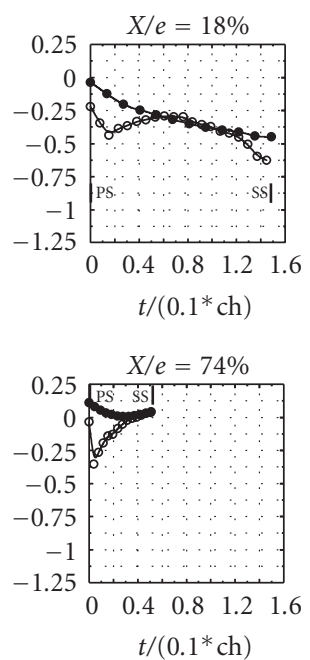

UNS rotor,

$\tau / \mathrm{ch}=2.7 \%, \phi=0.71$

- Along endwall

$\rightarrow$ Along blade tip

(b)
UNS rotor, $\tau / \mathrm{ch}=2.7 \%, \phi=0.5$

$\longrightarrow$ Along endwall

$\rightarrow$ Along blade tip
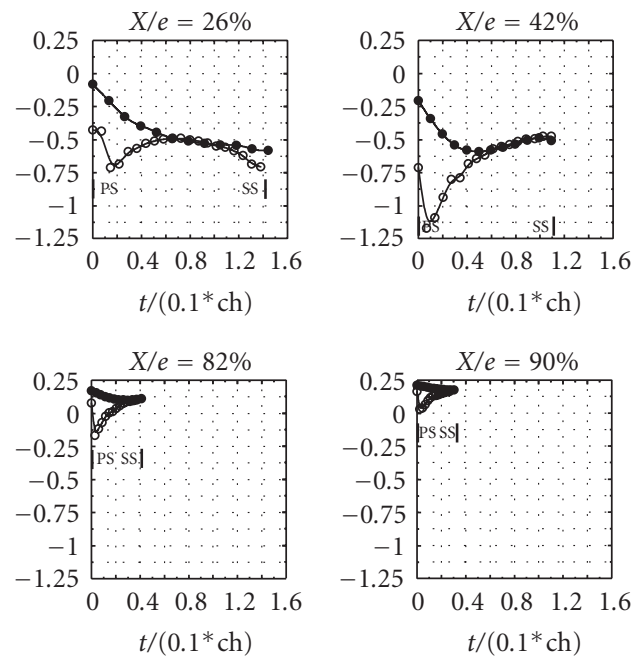

UNS rotor, $\tau / \mathrm{ch}=2.7 \%, \phi=0.71$

$\longrightarrow$ Along endwall $\rightarrow$ Along blade tip
UNS rotor, $\tau / \mathrm{ch}=2.7 \%, \phi=0.5$

$\longrightarrow$ Along endwall

$\rightarrow$ Along blade tip

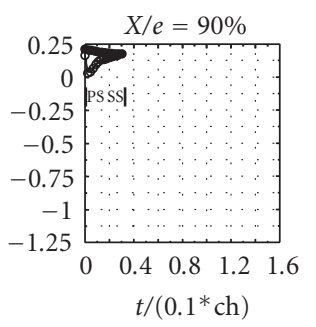

UNS rotor, $\tau / \mathrm{ch}=2.7 \%, \phi=0.71$

$\rightarrow$ Along endwall

$\rightarrow$ Along blade tip

FIGURE 9: Endwall and blade tip surface static pressure.

2.4.2. At $\tau / c h=2.7 \%$. When the clearance is increased, low pressure regions turned wider due to increased leakage and large tip vortex structure. It is reported in the previous discussions that effect of incidence on shifting the pressure trough is less obvious. But incidence is observed to affect the leakage direction. In the case of AXS rotors (low incidences) followed by TCS rotors, tip flow is less inclined to the camber line (less aligned to normal to the chord) when compared with the UNS rotor case. This effect of incidence on leakage direction is true at higher flow coefficient as well (lower incidence), where leakage is found almost tangential.

\section{Summary and Conclusions}

Results of the detailed computational investigation with three different sweep and tip clearance configurations are presented.

Observations near the tip reveal that UNS rotor shows very high sensitivity in the static pressure distribution for the tip clearance flows while AXS rotor is found to be stabler than the other rotors when the tip clearance is increased. Besides, AXS rotor has high loading capability and less tip clearance effect on blade loading at the near stall mass 

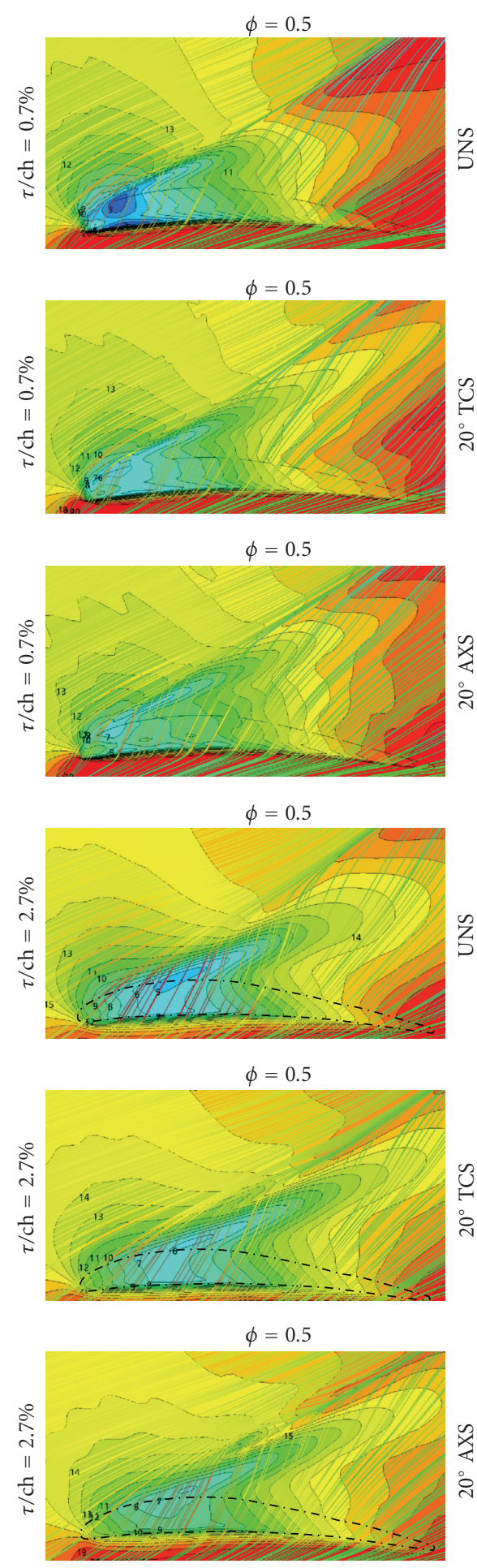
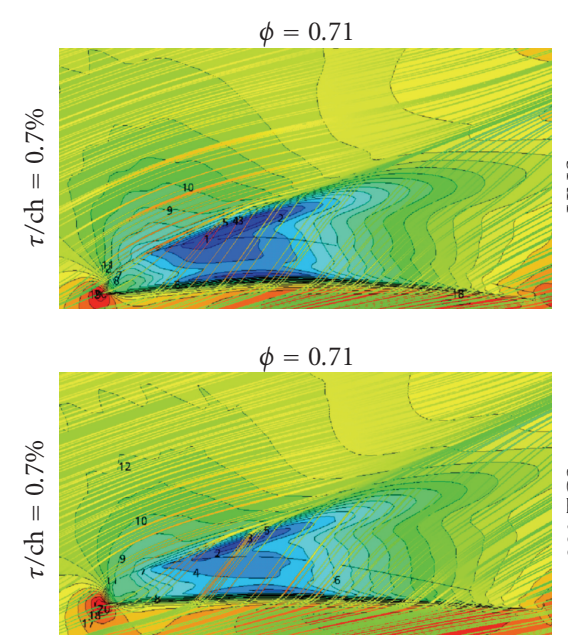

$\phi=0.71$

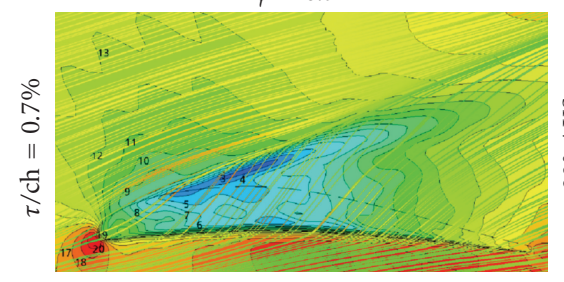

$\phi=0.71$

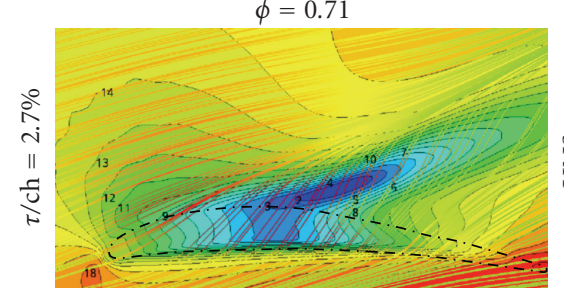

$\phi=0.71$

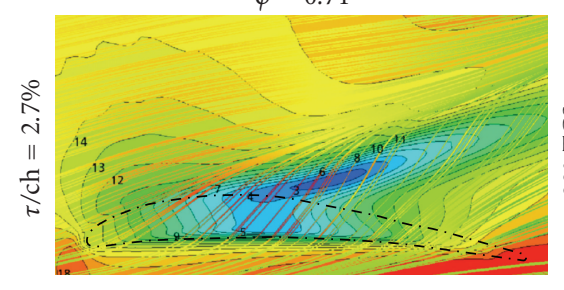

$\phi=0.71$

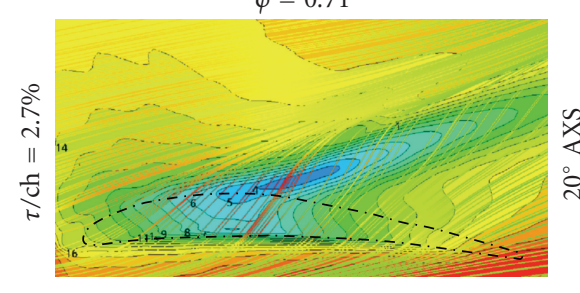

$\psi_{s}$

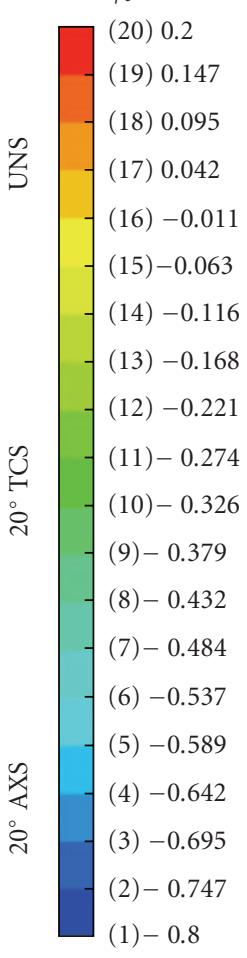

$\psi_{s}$

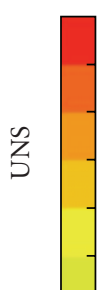

20) 0.2

(19) 0.147

(18) 0.095

(17) 0.042

(16) -0.011

(15) -0.063

(14) -0.116

(13) -0.168

(12) -0.221

(11) -0.274

(10) -0.326

(9) -0.379

(8) -0.432

(7) -0.484

(6) -0.537

(5) -0.589

(4) -0.642

(3) -0.695

$(2)-0.747$

(1) -0.8

Figure 10: Total pressure coefficient variation with streamline pattern on planes at mid tip gap height.

flow. Peak $C_{\Delta P}$, corresponding to the lowest static pressure trough seen on the rotor tip pressure distribution contours, is observed, which is caused by the leakage flow taking high curvature while entering the tip gap and forcibly spilling out to the other side through the gap. A downstream shift of the vortex rollup along the chord is observed with increased flow coefficient and increase in the tip gap height. In particular, the effect of flow coefficient is more predominant on the location of the pressure trough. The effect of the streamwise pressure gradient on the pressure side is found to have 
influence, to some extent, in picking the chordwise location for the pressure trough, but its influence on the leakage flow direction is less obvious. At small tip clearance $(\tau / \mathrm{ch}=0.7 \%)$, rotors did not show any constancy in the peak $C_{\Delta P}$ value with increasing flow coefficient, which indicates that the leakage did not "stabilize" for the local aerofoil loading, which is the case when the clearance is further increased to $\tau / \mathrm{ch}=$ $2.7 \%$.

Much of the leakage flow exits the clearance gap with a negative axial velocity component, creating vortex-related flow blockage. At both nonzero clearance levels, this blockage is the highest for the UNS rotor and the lowest for the AXS rotor. UNS rotor leakage vortices penetrated more into the passage before the axial velocity deficit gets sufficiently dissolved. This problem is found to be less for the swept rotors.

Flow mixing within the tip gap is observed at all chordwise locations including the highest $t / \tau(\approx 5.3)$ chordwise location as well as lowest $t / \tau(\approx 1.41)$ chordwise sections. Among the rotors, AXS rotor is found to incur low total pressure losses attributable to tip leakage. Incidence is found to influence tip flow leakage direction. In the case of AXS rotors (low incidences) followed by TCS rotors, tip flow is less inclined to the camber line when compared with the UNS rotor case. This effect of incidence on leakage direction is true at higher flow coefficient as well (lower incidence), where leakage is found almost tangential.

\section{Nomenclature}

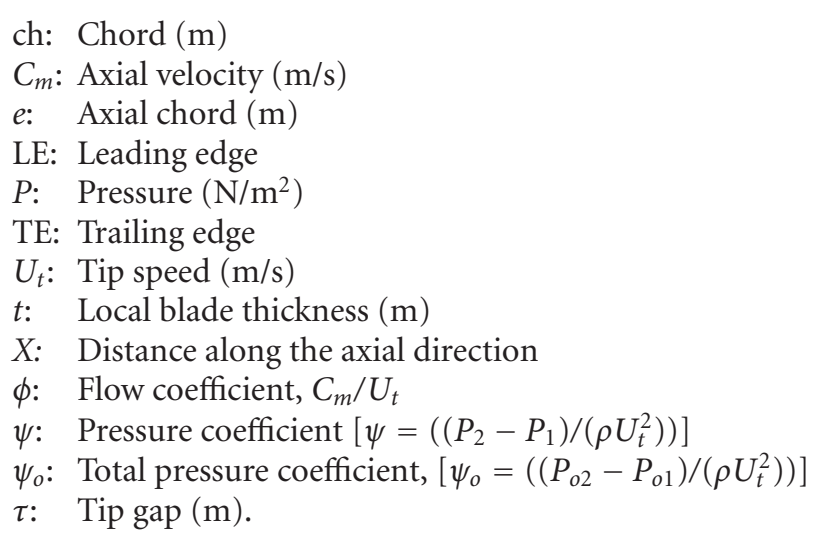

\section{Subscripts}

1: Inlet to rotor

2: Exit of rotor

o: Total

s: Static.

\section{References}

[1] P. V. Ramakrishna and M. Govardhan, "Study of sweep and induced dihedral effects in subsonic axial flow compressor passages-part I: design considerations-changes in incidence, deflection, and streamline curvature," International Journal of Rotating Machinery, vol. 2009, Article ID 787145, 11 pages, 2009.

[2] P. V. Ramakrishna and M. Govardhan, "ombined effects of forward sweep and tip clearance on the performance of axial flow compressor stage," in Proceedings of the ASME Turbo Expo, Orlando, Fla, USA, 2009, GT2009-59840.

[3] S. J. Gallimore, J. J. Bolger, N. A. Cumpsty, M. J. Taylor, P. I. Wright, and J. M. M. Place, "The use of sweep and dihedral in multistage axial flow compressor blading-part 1: university research and methods development," Journal of Turbomachinery, vol. 124, no. 4, pp. 521-532, 2002.

[4] G. S. McNulty, J. J. Decker, B. F. Beacher, and S. A. Khalid, "The impact of forward swept rotors on tip clearance flows in subsonic axial compressors," Journal of Turbomachinery, vol. 126, no. 4, pp. 445-454, 2004.

[5] V. M. Lei, Z. S. Spakovszky, and E. M. Greitzer, "A criterion for axial compressor hub-corner stall," Journal of Turbomachinery, vol. 130, no. 3, Article ID 031006, 10 pages, 2008.

[6] K. L. Suder and M. L. Celestina, "Experimental and computational investigation of the tip clearance flow in a transonic axial compressor rotor," in Proceedings of the International Gas Turbine and Aeroengine Congress and Exposition, pp. 1-15, June 1994, NASA TM-106711.

[7] M. Govardhan, B. H. L. Gowda, and R. M. Wankhade, "Experimental study of endwall and tip clearance flows in a two-dimensional turbine rotor blade cascade-effect of incidence angle," Journal of Thermal Science, vol. 9, no. 1, pp. 63-76, 2000.

[8] M. Govardhan, S. S. S. R. K. Sastri, and V. S. Vishnubhotla, "Experimental investigations of the three-dimensional flow in a large deflection turbine cascade with tip clearance," Journal of Thermal Science, vol. 7, no. 3, pp. 149-164, 1998.

[9] J. A. Storer and N. A. Cumpsty, "An approximate analysis and prediction method for tip clearance loss in axial compressors," Journal of Turbomachinery, vol. 116, no. 4, pp. 648-656, 1994.

[10] B. T. Sirakov and C. S. Tan, "Effect of unsteady stator wakerotor double-leakage tip clearance flow interaction on timeaverage compressor performance," Journal of Turbomachinery, vol. 125, no. 3, pp. 465-474, 2003.

[11] F. J. G. Heyes and H. P. Hodson, "Measurement and prediction of tip clearance flow in linear turbine cascades," Journal of Turbomachinery, vol. 115, no. 3, pp. 376-382, 1993.

[12] S. Kang and C. Hirsch, "Tip leakage flow in linear compressor cascade," Journal of Turbomachinery, vol. 116, no. 4, pp. 657664, 1994.

[13] J. Moore and J. S. Tilton, "Tip leakage flow in a linear turbine cascade," Journal of Turbomachinery, vol. 110, no. 1, pp. 18-26, 1988.

\section{Superscripts}

-: Circumferentially mass averaged

$=$ : Circumferentially and radially mass averaged. 

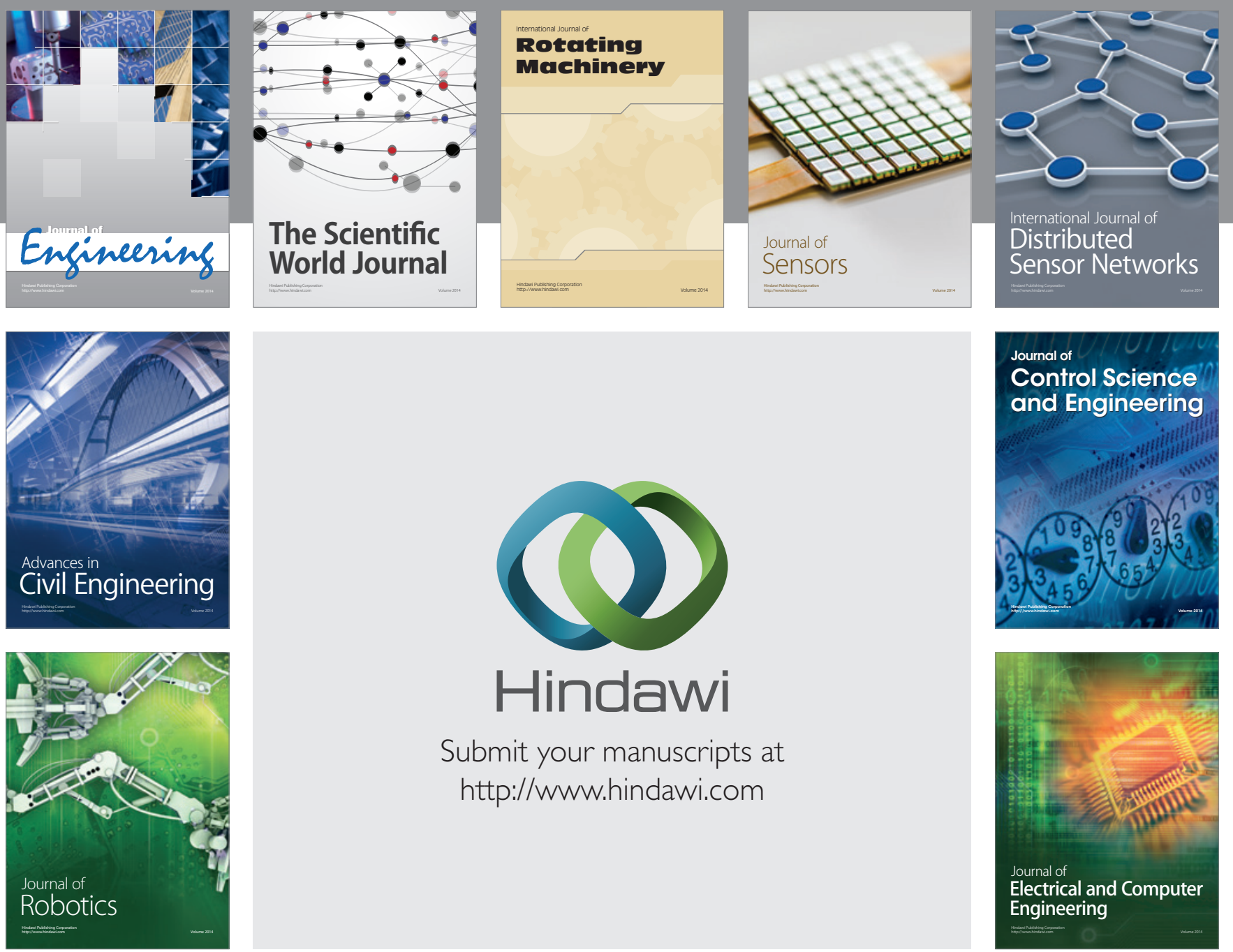

Submit your manuscripts at

http://www.hindawi.com
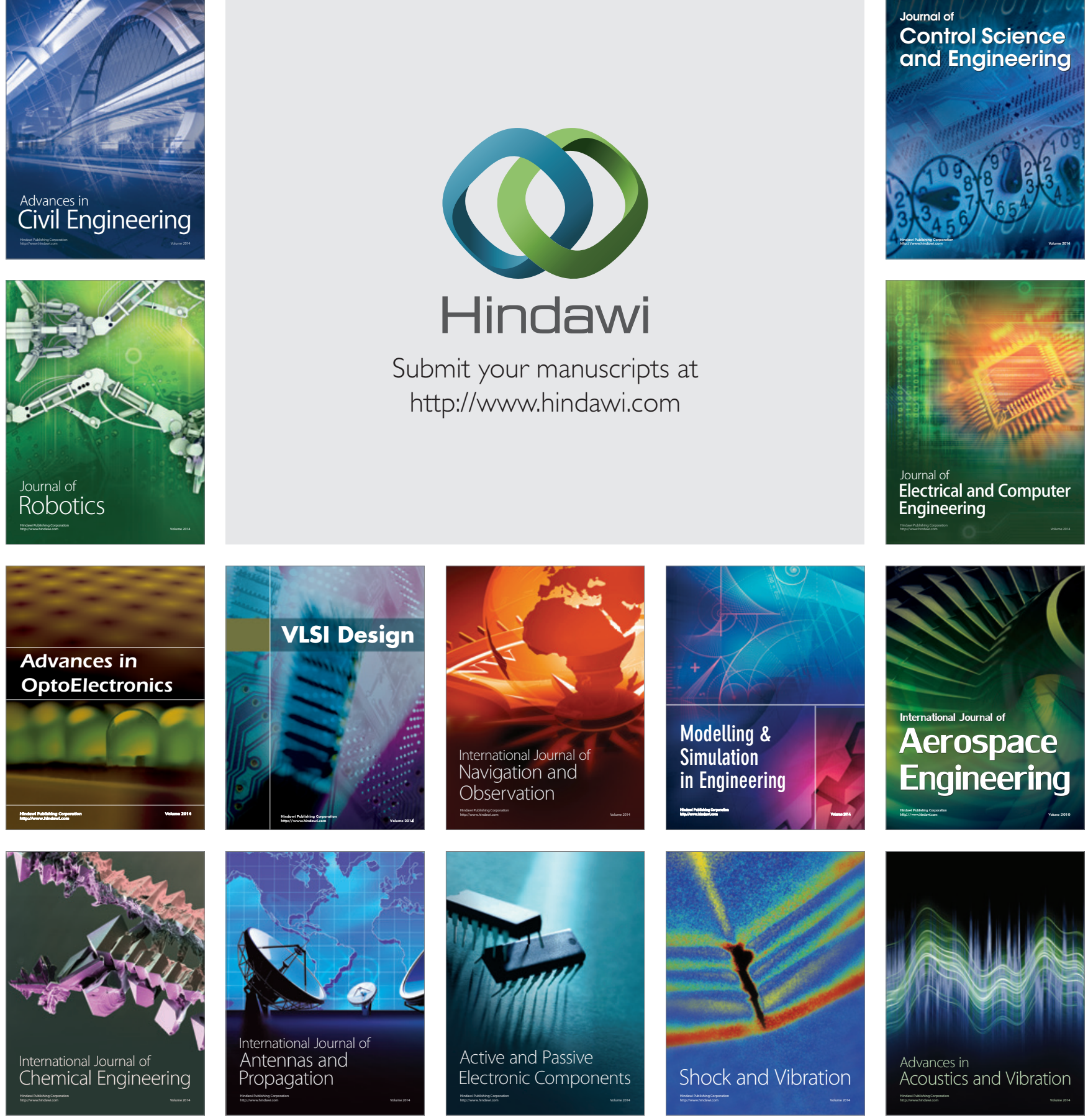